\title{
Recent advances in intelligent paradigms fusion and their applications
}

\author{
Lakhmi C. Jain $^{\mathrm{a}}$, Chee Peng Lim ${ }^{\mathrm{b}}$ and Ngoc Thanh Nguyen ${ }^{\mathrm{c}}$ \\ ${ }^{a}$ University of South Australia, Australia \\ E-mail:LLakhmi.Jain@unisa.edu.au \\ ${ }^{\mathrm{b}}$ University of Science Malaysia, Malaysia \\ ${ }^{\mathrm{c}}$ Wroctaw University of Technology, Poland
}

Over the past decades, computerized intelligent paradigms have attracted much attention from researchers. Advances in intelligent techniques and approaches, which include neural networks, knowledgebased systems, fuzzy logic, evolutionary algorithms, agent-based techniques, case based reasoning etc, have resulted in many successful applications of these innovative systems. Indeed, applicability of intelligent paradigms to many diverse domains have been demonstrated, which include pattern recognition, image and signal processing, control and automation, robotics, data mining, biomedicine, knowledge management, finance and banking.

Instead of employing a single intelligent paradigm, one recent trend of research and development is on the fusion of different intelligent paradigms to tackle complex problems. The combination of more than one intelligent techniques can be realized in many forms, either by a modular cooperation of two (or more) intelligent techniques, each with its own identity; or by fusing one technique into another to form an integrated system. The main rationale behind intelligent paradigms fusion is to exploit the advantages of each intelligent technique and, at the same time, to avoid its limitations, for solving a particular problem.

In this special issue, a number of articles which address the usefulness of different intelligent paradigms and their fusions are presented. The intelligent paradigms investigated include fuzzy models, evolution- ary algorithms, multi-agent systems, neural networks, particle swam optimization techniques, and artificial immune systems. Applications of these systems are demonstrated in various fields, ranging from real estate appraisal, e-learning, sensor networks, to biomedical data analysis. A summary of the articles covered in this special issue is as follows.

Fuzzy models have emerged as an effective tool for intelligent data analysis, where the data can be both quantitative and qualitative. In Król et al., the TakagiSugeno-Kang (TSK) fuzzy model is optimized using evolutionary algorithms. The resulting model is applied to real estate appraisals. Two approaches are examined. The first focuses on learning the rule base while the second deals with learning the rule base and tuning the membership functions simultaneously. The evolutionary algorithms are based on the Pittsburgh approach with real-valued chromosomes comprising the whole rule base or both the rule base and parameters of the membership functions. Applicability of the system is evaluated using a total of 150 real sales transactions made in one of Polish cities.

Development in innovative computer technologies has offered many promises in the field of education, especially in distance learning. However, a crucial issue in educational technology is the provision of instructional environments and conditions that comply with different educational goals and learning abilities. In Serçe et al., an Adaptive Intelligent Learning Sys- 
tem (AILS) is designed for use with generic Learning Management System (LMS). Developed based on the multi-agent system technology, the AILS aims to provide e-learning services to online learners. It has the ability to identify and monitor a learner's learning process according to the learner's profile. A demonstration is also presented to illustrate the functionality of the AILS.

Sensor networks are a practical tool in many areas, which include monitoring real-life phenomena, traffic control, and industrial applications. Sensor data, however, are often noisy, incomplete, and unreliable. In Petrosino \& Staiano, the Adaptive Neural-Fuzzy Inference System (ANFIS) is used for sensor data cleansing. The objective is to reduce the uncertainty associated with sensor data in order to obtain a more accurate estimate of sensor readings. Experiments using a public domain sensor data set comprising temperature, light, voltage, and humidity readings are conducted. The results from three methods, i.e., ANFIS, a polynomial regression kernel, and Kalman filtering, are compared, with their merits discussed.

Protein is part and parcel of every cell in an organism. In Datta et al., a new approach that combines artificial neural network and particle swarm optimization techniques for predicting three dimensional tertiary structure of protein is presented. The Ab-initio approach is used for global minimization of the energy function. By finding the main chain dihedral angles corresponding to the global minima of the CHARMM energy function using particle swarm optimization, the proposed approach is able to predict the native structure of protein. The side chain dihedral angles are predicted using a three-layered artificial neural network. The approach is able to reduce the dimensionality of the search space, and has been shown to outperform a number of classical techniques in protein structure prediction.

Breast cancer is one of the most commonly diagnosed cancers for women. In Menolascina et al., how an intelligent fusion paradigm can be employed in the elucidation of biological dynamics of cancerous processes is investigated. Specifically, the Induction of Fuzzy Rules with Artificial Immune Systems

(IFRAIS) is used for mining the array CGH data and for extracting useful knowledge in experimental oncology. The main advantage of IFRAIS is that it is able to discover fuzzy classification rules that are easily comprehensible to domain users. The results are validated from both statistical and biological perspectives in order to ascertain the usefulness of IFRAIS in breast cancer familiarity profiling.

The guest editors would like to express sincere gratitude to authors who have contributed their articles to this special issue. The guest editors would also like to thank the reviewers for reviewing the articles and for suggesting constructive comments to improve quality of the articles. Appreciation is also extended to the journal production team for the help and support in producing this special issue. 